\title{
The conserved function of Skp1 in meiosis
}

\author{
Josh D. McLoud and Ming Yang* \\ Department of Botany, Oklahoma State University, Stillwater, OK, USA \\ ${ }^{*}$ Correspondence: ming.yang@okstate.edu \\ Edited by: \\ Xiaogang Wu, Indiana University-Purdue University Indianapolis, USA \\ Reviewed by: \\ Xiaogang Wu, Indiana University-Purdue University Indianapolis, USA \\ Sudipto Saha, Case Western Reserve University, USA \\ Mingyi Wang, The Samuel Roberts Noble Foundation, USA \\ Fan Zhang, Mount Sinai School of Medicine, USA
}

\section{A commentary on}

SCF ensures meiotic chromosome segregation through a resolution of meiotic recombination intermediates

by Okamoto, S.-Y., Sato, M., Toda, T., and Yamamoto, M. (2012). PLoS One 7, e30622. doi: 10.1371/journal.pone.0030622

Many components of regulatory networks governing basic cellular functions are highly homologous in diverse species. An important question is how such conservation of individual components is translated into the conservation at the level of regulatory networks. An intriguing case regarding this question is concerned with the function of the Skp1 protein in meiosis. Skp1 is a conserved subunit of the Skp1-Cullin-F-boxprotein (SCF) E3 ubiquitin ligase (Tyers and Jorgensen, 2000; Willems et al., 2004). For nearly 13 years, a distinct meiotic phenotype was described only in a null mutant of Skp1 in Arabidopsis thaliana, namely arabidopsis-skp1-like1-1 (ask1-1) (Yang et al., 1999; Yang and Ma, 2001; Wang et al., 2004; Wang and Yang, 2006). It raises the question of whether such a phenotype indicates a plant-specific function of Skp1 (or the corresponding SCF) in meiosis. However, a strikingly similar meiotic phenotype was recently reported by Okamoto et al. (2012) in skp1 mutants of fission yeast (Schizosaccharomyces pombe). These phenotypic similarities suggest the presence of a conserved network involving an SCF that regulates the progression of recombination in meiosis.

The first similarity is that the numbers of spores produced by a meiocyte range from two to more than four spores in ask1-1 (Yang et al., 1999) and the yeast $s k p 1$ mutants. In many meiotic mutants, the meiotic products produced by a meiocyte are either more or fewer than the normal four spores. The wide range of meiotic product number per meiocyte places ask1-1 and the yeast $s k p 1$ mutants in a rare group. The second similarity is indicated by the morphology and behavior of chromosomes during meiosis I. Chromosomes entangle, non-disjoin, are stretched by the anaphase spindle, and as a result, are unevenly distributed to meiotic products in both ask1-1 (Yang et al., 1999; Wang et al., 2004) and the yeast skp1 mutants. Chromosomes are asynaptic in prophase I in ask1-1 (Wang et al., 2004). Fission yeast normally undergoes homologous chromosome pairing instead of synapsis in prophase I. It is unknown whether the homologous chromosome pairing in the yeast $s k p 1$ mutants is also affected, but it may be so based on the overall similarities in chromosome behavior between ask1-1 and the yeast skp1 mutants. Spindle behavior is intimately linked to chromosome behavior. It is unsurprising that the spindle seems similarly affected by chromosome entanglement in ask1-1 and the yeast skp1 mutants. Although the experimental approaches somewhat differ in the investigations of the two organisms - study of still images in Arabidopsis and study of timelapsed videos in yeast, similar conclusions can be made on the impact of entangled chromosomes on the spindle morphology. The Arabidopsis work found anaphase spindles that were either shorter than metaphase spindles or longer than typical anaphase spindles in ask1-1 (Yang and Ma, 2001). The yeast work revealed bending, collapsing, reforming, and re-elongation of spindles. It can be said that spindles are deformed in a similar fashion when they struggle to separate entangled chromosomes in the skp1 mutants of both organisms. Lastly, the underlying cause of the chromosome entanglement may be attributed to the presence of unresolved recombination intermediates in ask $1-1$ and the yeast $s k p 1$ mutants. In ask1-1, electrondense materials abnormally accumulate on chromosomes in prophase I (Wang et al., 2004), the dynamics of a cohesin on meiotic chromosomes is altered (Zhao et al., 2006), and recombination is promoted in plants heterozygous for ask1-1 (Wang and Yang, 2006). In comparison, several proteins involved in recombination are retained longer than usual on meiotic chromosomes in the yeast $s k p 1$ mutants. Thus, evidence from both Arabidopsis and yeast suggests that Skp1 is involved in promoting the resolution of recombination intermediates in meiosis. Perhaps a moderate reduction in the ASK1 function in Arabidopsis plants heterozygous for ask1-1 enhances the formation of recombination intermediates not to a toxic level so that recombination frequency is increased.

The Skp1 protein is not expected to be directly involved in the recombination process, and yet the phenotypes of its Arabidopsis and yeast mutants closely resemble each other. It is also noted that the yeast work was carried out without the knowledge of the Arabidopsis work (The Arabidopsis work was not cited by the yeast paper). The converging evidence from both Arabidopsis and yeast suggests the existence of a conserved network in the regulation of recombination progression in meiosis. An F-box protein with a DNA helicase domain is suggested by Okamoto et al. (2012) to be the one linking Skp1 to the recombination process in yeast. The Arabidopsis genome does not contain a homolog of the yeast F-box-associated DNA helicase (based on BLAST search using the yeast protein sequence as query) although it has many proteins containing either an F-box or a DNA helicase domain. 
Further elucidation of the predicted network in diverse species will likely reveal novel aspects in the regulation of meiotic recombination.

\section{ACKNOWLEDGMENT}

This work was supported, in part, by the NSF Bridge to the Doctorate Fellowship through OK-LSAMP to Josh D. McLoud.

\section{REFERENCES}

Okamoto, S.-Y., Sato, M., Toda, T., and Yamamoto, M. (2012). SCF ensures meiotic chromosome segregation through a resolution of meiotic recombination intermediates. PLoS One 7, e30622. doi: 10.1371/ journal.pone.0030622

Tyers, M., and Jorgensen, P. (2000). Proteolysis and the cell cycle: with this RING I do thee destroy. Curr. Opin. Genet. Dev. 10, 54-64.
Wang, Y., Wu, H., Liang, G., and Yang, M. (2004). Defects in nucleolar migration and synapsis in male prophase I in the ask1-1 mutant of Arabidopsis. Sex. Plant Reprod. 16, 273-282.

Wang, Y., and Yang, M. (2006). The ARABIDOPSIS SKP1-LIKE1 (ASK1) protein acts predominately from leptotene to pachytene and represses homologous recombination in male meiosis. Planta 223, 613-617.

Willems, A. R., Schwab, M., and Tyers, M. (2004). A hitchhiker's guide to the cullin ubiquitin ligases: SCF and its kin. Biochim. Biophys. Acta 1695, 133-170.

Yang, M., Hu, Y., Lodhi, M., McCombie, R. W., and Ma, H. (1999). The ARABIDOPSIS SKP1-LIKE1 gene is essential for male meiosis and may control homologue separation. Proc. Natl. Acad. Sci. U.S.A. 96, 11416-11421.

Yang, M., and Ma, H. (2001). Male meiotic spindle lengths in normal and mutant Arabidopsis cells. Plant Physiol. $126,622-630$.
Zhao, D., Yang, X., Quan, L., Timofejeva, L., Rigel, N. W., Ma, H., and Makaroff, C. A. (2006). ASK1, a SKP1 homolog, is required for nuclear reorganization, presynaptic homolog juxtaposition and the proper distribution of cohesin during meiosis in Arabidopsis. Plant Mol. Biol. 62, 99-110.

Received: 07 August 2012; accepted: 26 August 2012; published online: 14 September 2012.

Citation: McLoud JD and Yang M (2012) The conserved function of Skp1 in meiosis. Front. Gene. 3:179. doi: 10.3389/fgene.2012.00179

This article was submitted to Frontiers in Systems Biology, a specialty of Frontiers in Genetics.

Copyright (C) 2012 McLoud and Yang. This is an openaccess article distributed under the terms of the Creative Commons Attribution License, which permits use, distribution and reproduction in other forums, provided the original authors and source are credited and subject to any copyright notices concerning any third-party graphics etc. 